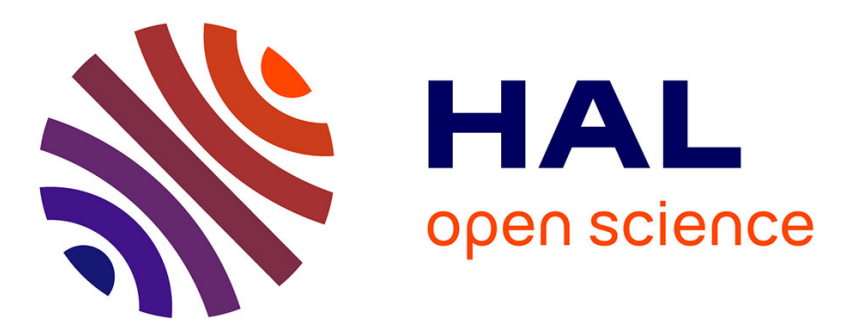

\title{
Laser-Induced Damage in Silica Nanofibers in Air and Immersed in Different Liquids in the Nanosecond Regime
}

\author{
Maha Bouhadida, Pierre-Enguerrand Verdier, Sylvie Lebrun
}

\section{To cite this version:}

Maha Bouhadida, Pierre-Enguerrand Verdier, Sylvie Lebrun. Laser-Induced Damage in Silica Nanofibers in Air and Immersed in Different Liquids in the Nanosecond Regime. IEEE Photonics Technology Letters, 2021, 33 (17), pp.967-970. 10.1109/LPT.2021.3101438 . hal-03359750

\section{HAL Id: hal-03359750 \\ https://hal-iogs.archives-ouvertes.fr/hal-03359750}

Submitted on 30 Sep 2021

HAL is a multi-disciplinary open access archive for the deposit and dissemination of scientific research documents, whether they are published or not. The documents may come from teaching and research institutions in France or abroad, or from public or private research centers.
L'archive ouverte pluridisciplinaire HAL, est destinée au dépôt et à la diffusion de documents scientifiques de niveau recherche, publiés ou non, émanant des établissements d'enseignement et de recherche français ou étrangers, des laboratoires publics ou privés. 


\title{
Laser-Induced Damage in Silica Nanofibers in Air and Immersed in Different Liquids in the Nanosecond Regime
}

\author{
Maha Bouhadida, Pierre-Enguerrand Verdier, Sylvie Lebrun
}

\begin{abstract}
Thanks to their unique optical properties, silica nanofibers are used in a growing number of applications. Moreover, the possibility of immerging them in various media adds another interesting feature to these devices. However, as for all optical components, nanofibers are limited by the optical damage that can be induced by a laser. In this Letter, we present for the first time to our knowledge Laser-Induced Damage Threshold (LIDT) measurements of silica nanofibers in air and in three common liquids (ethanol, isopropanol, and water). The experiments were performed in the nanosecond regime with a pump source emitting at the wavelength of $532 \mathrm{~nm}$. Different nanofiber radii (from $220 \mathrm{~nm}$ to $450 \mathrm{~nm}$ ) and two nanofiber lengths $(2 \mathrm{~cm}$ and $8 \mathrm{~cm})$ were tested. We firstly present the experimental setup and protocol. A significant number of samples were realized, and the results were highly repeatable. We also showed that immerging a nanofiber in a liquid substantially enhanced the LIDT, the most important increase having been obtained for nanofibers immersed in water for which the LIDT energy was almost multiplied by a factor of 2 compared with air $(0.38 \mu \mathrm{J}$ in air vs $0.71 \mu \mathrm{J}$ in water for a radius of $350 \mathrm{~nm})$. This property offers a new degree of freedom to widen the field of applications of nanofibers, where high peak powers are needed.
\end{abstract}

Index Terms-Laser-induced damage, silica nanofibers, nonlinear applications

\section{INTRODUCTION}

$\mathrm{O}$ ptical tapered nanofibers are fabricated by pulling standard silica fibers until their diameters become typically smaller than $1 \mu \mathrm{m}$. The uniform part, called the nanofiber, is linked to the untapered end-fibers via two flared sections called the tapers and can be inserted in fibered networks with very low losses. These components present unique optical properties that make them very attractive for a growing number of applications. Indeed, at such small diameters, there is a strong confinement of light inside the nanofiber: peak power densities of several tens of $\mathrm{GW} / \mathrm{cm}^{2}$ can be reached enabling the generation of efficient nonlinear effects [1]. Nanofibers can also exhibit an intense evanescent field. As an example, for a radius of $200 \mathrm{~nm}$ and at the wavelength of $532 \mathrm{~nm}$, the fraction of the evanescent field of the optical mode is $20 \%$ in air and can increase to $60 \%$ when immersed in water. This property has been exploited for optical sensing [2] or optical traps for quantum applications [3]. The excitation of optical nonlinearities such as Stimulated Raman Scattering (SRS) [4] or Kerr effect in the evanescent field of nanofibers immersed in liquids has also been demonstrated [5].

As it is the case for all optical dielectric components, a limitation of nanofibers is the optical damage that can be induced by a laser. In the ns regime and for dielectric materials, the origin of these damages is the laser absorption by defects on the surface (microcracks or pollutants) that generates high pressure and temperature that can lead to a micro explosion [6]. In silica bulk optics and fibers, these damages, obtained for fluence values between a fraction of $\mathrm{J} / \mathrm{cm}^{2}$ and several $\mathrm{kJ} / \mathrm{cm}^{2}$ $[7,8,9]$, are partially destructive and gradually degrade the performances of the components. In nanofibers, the issue is more radical: the damage is immediately followed by the rupture of the nanofiber, which is just broken in two, leading to an irreversible and fully destructive effect. In the following the energy per pulse that is necessary to break the nanofiber will be called the Laser-Induced Damage Threshold (LIDT) energy.

The optical damage of nanofibers is evoked in quantum optics applications that are performed in vacuum in the continuous wave regime. The injected power is generally limited to several tens of $\mathrm{mW}$ [10]. In nonlinear optics, experiments using nanofibers are generally performed in air, gas, or liquids as the surrounding medium. Contrary to vacuum, the more efficient heat transfer from the nanofiber to the external medium enables to use much higher powers. The knowledge of the LIDT energy is crucial for the design of nanofiber-based components to predict whether the threshold of the nonlinear effect will be reached before the breaking point. However, for pulsed regimes, there is a lack of experimental data on the LIDT energy in nanofibers in the literature. In this letter we present some first experimental data on the LIDT energy of silica nanofibers in ambient air and immersed in different low-index common liquids, in the ns regime where compact, low-cost and reliable laser sources are available. 


\section{EXPERIMENTAL SETUP AND PROTOCOL}

The experimental setup is shown in Fig. 1. The preparation and the drawing of the nanofibers were performed in a class-5 clean room. In the following experiments, we have used the SMF28 fiber (ref. Corning). As this fiber is highly multimode at $532 \mathrm{~nm}$ which is the pump wavelength and as the beam quality is an important parameter for these damage studies, high-order modes were filtered beforehand by pulling the input connecting fiber over $1 \mathrm{~cm}$ until reaching a core radius of $1.3 \mu \mathrm{m}$ and a cladding radius of $20 \mu \mathrm{m}$ allowing the propagation of the fundamental mode only (single mode filter on Fig. 1). We have used short and straight connecting fibers to remain on a single mode propagation from the input (indicated by $\mathrm{E}_{1}$ ) to the output (indicated by $E_{\text {out }}$ ). In Fig. 1 (bottom right) we show the beam profile at the output, with its cross-section showing a nearly Gaussian profile. Then, before being pulled the fiber was removed from its polymer cladding with a mechanical stripper and cleaned with an optical paper wet by a drop of ethanol. The nanofibers were drawn with a pulling rig using a simple butane flame [4]. The butane flame softens the fiber central part while two computer-controlled translation stages elongate it following the "pull and brush" technique to create the nanofiber and the tapers. In a few words, the pulling process is divided into cycles. Each cycle adds a small section to the taper while the radius of the fiber cladding is reduced by a constant ratio to reach the nanofiber waist radius at the end of the last cycle. At cycle iteration number $\mathrm{n}$, the heat zone length is chosen as a power law of $\mathrm{n}$, enabling to create few $\mathrm{cm}$ length tapers. Once drawn, and before being transported to the experimental set-up out of the clean room, the nanofiber was put in a tank with a removable protection glass cover. The pump source beam (ref. Horus HLX-G-F004, $\lambda=532 \mathrm{~nm}$, frequency repetition $\mathrm{f}=$ $4.7 \mathrm{kHz}$, pulse width duration (FWHM) $\Delta \mathrm{t}_{1 / 2}=900 \mathrm{ps}$, $\mathrm{E}_{\max }=10 \mu \mathrm{J}$, linearly polarized) was sent on a half-wavelength plate associated with a polarizer cube to control the incident energy on the nanofiber. The rejected beam served as a reference for the input energy ( $\left.\mathrm{E}_{\text {ref }}\right)$. The direct beam was launched in the SMF28 fiber through a microscope objective, propagated across the single mode filter, the nanofiber and was collected at the output for energy measurements. The input energy $E_{1}$ just after the single mode filter was calibrated with respect to $E_{\text {ref. }}$. The nanofiber was a little bit stretched to be straight but not constrained and kept in the position thanks to two smooth magnets. The pump beam was injected in the fiber at low energy and the optical transmission was optimized. For experiments with liquids, the tank was filled carefully with a micro-syringe until the nanofiber was totally immersed. The laser input energy was slowly increased until the nanofiber broke by step of $0.02 \mu \mathrm{J}$ for low input energies then by step of $0.01 \mu \mathrm{J}$ or even less when we were close to the LIDT energy. Input and output energies were recorded during all the process. We observed just before the LIDT energy heralding fluctuations in light intensity in the first half of the nanofiber where was localized the point at which the nanofiber broke. Once theses fluctuations had been observed a very small increase of the input energy induced the rupture of the nanofiber.

Four external media with different refractive index were used: ambient air $(\mathrm{n}=1)$, tap water $(\mathrm{n}=1.33)$, ethanol $(\mathrm{n}=1.36)$, and isopropanol $(\mathrm{n}=1.38)$. Three different nanofiber radii were tested: $220 \mathrm{~nm}, 300 \mathrm{~nm}, 350 \mathrm{~nm}$ (respectively denoted by NF1, NF2 and NF3). The length of each nanofiber was $2 \mathrm{~cm}$. To test the repeatability of the measurements, we have drawn 10 nanofibers of each radius for each medium.

\section{RESULTS AND DISCUSSION}

The transmission $\mathrm{T}$ of the immersed nanofibers with the tapers, defined as $T=E_{\text {out }} / E_{1}$, remained very stable during the whole experiment until reaching the LIDT energy (i.e. for a duration of about 30 minutes) with an average value around $90 \%$. This was not the case when they were in air: the average transmission (calculated over all the samples) dropped from $80 \%$ to $57 \%$ during the experiment, which was attributed to dust deposition.

We have plotted in Fig. 2 the incident LIDT energy inside the nanofiber, named $E_{i n}$, for each radius $r_{N F}$ and each external medium. As the tapers were symmetrical, $E_{i n}$ was defined as $E_{\text {in }}=\eta \sqrt{T} E_{1} . \eta$ is the fractional energy inside the nanofiber and is defined as: $\eta=\frac{\int_{0}^{2 \pi} \int_{0}^{r} N F(\vec{e} \times \vec{h}) \cdot \vec{z} r d r d \phi}{\int_{0}^{2 \pi} \int_{0}^{\infty}(\vec{e} \times \vec{h}) \cdot \vec{z} r d r d \phi} \cdot \vec{e}$ is the electrical field, $\vec{h}$ is the magnetic field, $\vec{z}$ is the direction of propagation, $r$ and $\phi$ are respectively the radial and azimuthal coordinates [11].

The standard deviation on the incident energies $E_{\text {in }}$ calculated on 10 nanofibers per measurement point was estimated to be $0.01 \mu \mathrm{J}$ in all cases, giving error bars smaller than the symbols. In this range of radii, the uncertainty on the radii was below 10 nm (see MEB pictures on Fig. 1). We also made one measurement in air and one in water for a larger nanofiber radius of $450 \mathrm{~nm}$ : the LIDT energy in air was $0.66 \mu \mathrm{J}$ and we were not able to reach it when immersed in water with the maximum available incident energy of $2 \mu \mathrm{J}$, giving a lower limit of the LIDT for this radius.

In Fig. 2 we note that for a given external medium the LIDT energy increases with the radius. As said in the introduction and as studied in $[10,12]$, the pump light is absorbed by surface pollutants. This generates the formation of a hot point hence an increase of the nanofiber temperature leading to a catastrophic effect responsible for its damage. This temperature is not precisely determined yet and is the object of further studies. However it has been calculated that for a given pump energy, the temperature of a nanofiber increases as its radius decreases [12]. This trend, demonstrated in vacuum, can be extrapolated to a nanofiber surrounded by a fluid and can explain this first observation. In Fig. 2 we also observe that the LIDT energy in liquids was always higher or equal to the one in air whatever the nanofiber radius. To explain these results we have plotted in Fig. 3, for the different refractive indexes of the studied external media, the amplitude of the z-component of the Poynting vector of the fundamental mode HE11, normalized 
with respect to the transported power, i.e. $S_{z}=$ $(\vec{e} \times \vec{h}) \cdot \vec{z}$ $\frac{(\vec{e} \times \hat{h}) \cdot \vec{z}}{\int_{0}^{2 \pi} \int_{0}^{\infty}(\vec{e} \times \vec{h}) \cdot \vec{z} r d r d \phi}$ [11]. For this illustration the chosen nanofiber radius is $220 \mathrm{~nm}$ but the mode profiles remain globally similar for the other studied radii, with a discontinuity at the interface typical of the vectorial mode HE11. We can see that the mode spreads over larger distances as the refractive index of the surrounding medium increases. We have also plotted in Fig. 4 the fractional energy inside the nanofiber $\eta$ versus the nanofiber radius for the different external media. The evanescent field can be important when the nanofiber is immersed in a liquid (typically between $20 \%$ and $70 \%$ as illustrated on Fig. 4 for the studied radii and liquids) and can also lead to an increase of the temperature of the liquid by visible residual absorption of the pump (low but existing, for example in ethanol the absorption coefficient is $1.5 \times 10^{-3} \mathrm{~cm}^{-1}$ at $532 \mathrm{~nm}$ [13]). The heating of the liquid by the evanescent field will lead to a less efficient cooling of the nanofiber. For a given radius, the fraction of energy in the nanofiber is much higher in air than in the liquids. At $220 \mathrm{~nm}$, the LIDT energy is nearly the same in air and in alcohols, meaning that the temperature achieved in the nanofiber is about the same. The thermal conductivity of alcohols (typically around $0.2 \mathrm{~W} / \mathrm{m} . \mathrm{K}$ ) is higher than the air's one $(0.03 \mathrm{~W} / \mathrm{m} . \mathrm{K})$ so we can expect at first sight a more efficient cooling of the nanofiber. However, this effect is counterbalanced by the heating of the alcohols by the evanescent field. At $350 \mathrm{~nm}$, as the fractional energy in the evanescent field decreases, the heating of the alcohols by the light is lower, enabling a more efficient cooling of the nanofiber by the alcohols, explaining a higher LIDT energy than in air. The thermal conductivity of water $(0.60 \mathrm{~W} / \mathrm{m} . \mathrm{K})$ being much higher than the alcohols and air ones, the balance is always in favor of an efficient cooling, explaining why the LIDT in water is always the highest. To confirm this interpretation, we measured the LIDT energy of a nanofiber having a radius of $350 \mathrm{~nm}$ and immersed in methanol (same refractive index as water, so same mode profile, but lower thermal conductivity) and obtain a LIDT energy of $0.50 \mu \mathrm{J}$ (instead of $0.71 \mu \mathrm{J}$ for water).

We also tested nanofibers having a length of $8 \mathrm{~cm}$ and immersed in ethanol and isopropanol. Under these conditions we have demonstrated in a previous work that SRS was efficiently excited in the liquid in the evanescent field [4]. However, as the nanofiber broke in its first half, at a point where SRS just begun to be excited, the contribution of the Stokes energy to the LIDT has a neglectable impact on the LIDT energy. We studied nanofibers with radii corresponding to NF1, NF2 and NF3. As shown in Table 1, very similar results were found, as expected. Indeed, as the ns pulse pump length was about $10 \mathrm{~cm}$, it covered all the nanofiber, and there is no influence of the length of the nanofiber on the LIDT energy in the range of a ten of $\mathrm{cm}$.

We also tested a single mode fiber (ref. 460HP from Thorlabs). The differences with the experiments using the SMF28 fiber were obviously the input and output fibers (in particular, no need of a single mode filter before the nanofiber) but also the tapers shapes which were adapted to this new fiber. In the nanofiber part, as the core was fully melted with the cladding, we can consider that there was almost no difference between the two standard fibers. The length of the nanofibers was $8 \mathrm{~cm}$. Ten samples of each radius $(220 \mathrm{~nm}, 300 \mathrm{~nm}$ and 350 $\mathrm{nm})$ were fabricated and immersed in ethanol. As shown in Table 1, and as expected, very similar results for LIDT energies were found with this fiber.

TABLE 1. COMPARISON OF THE LIDT INCIDENT ENERGIES E IN $(\mu \mathrm{J})$ INSIDE THE NANOFIBER FOR $L=2 \mathrm{CM}$ AND $L=8 \mathrm{CM}$ FOR NANOFIBERS IMMERSED IN ETHANOL AND ISOPROPANOL. TWO FIBERS WERE TESTED (SMF28 AND $460 \mathrm{HP})$.

\begin{tabular}{|c|c|c|c|c|c|}
\hline Radius & $\begin{array}{l}\mathrm{L}=2 \mathrm{~cm} \\
\mathrm{SMF} 28 \\
\text { ethanol }\end{array}$ & $\begin{array}{l}\mathrm{L}=8 \mathrm{~cm} \\
\text { SMF2 } \\
\text { ethanol }\end{array}$ & $\begin{array}{l}\mathrm{L}=2 \mathrm{~cm} \\
\text { SMF28 } \\
\text { isopropanol }\end{array}$ & $\begin{array}{l}\mathrm{L}=8 \mathrm{~cm} \\
\text { SMF28 } \\
\text { isopropanol }\end{array}$ & $\begin{array}{l}\mathrm{L}=8 \mathrm{~cm} \\
460 \mathrm{HP} \\
\text { ethanol }\end{array}$ \\
\hline $220 \mathrm{~nm}$ & 0.13 & 0.10 & 0.11 & 0.09 & 0.10 \\
\hline $300 \mathrm{~nm}$ & 0.30 & 0.32 & 0.27 & 0.28 & 0.29 \\
\hline $350 \mathrm{~nm}$ & 0.48 & 0.42 & 0.44 & $\begin{array}{l}\text { Not } \\
\text { measured }\end{array}$ & 0.45 \\
\hline
\end{tabular}

These data provide a first thumb of rule for the design and optimization of nanofibers for nonlinear applications where high peak powers are needed, by comparing the LIDT energy with the nonlinear threshold energy defined by $E_{t h} \approx$ $20 \Delta t_{1 / 2} / g_{N L} L$ where $g_{N L}$ is the modal nonlinear gain and $L$ is the nanofiber length [4]. As future studies, other temporal regimes (fs, ps, continuous-wave) should also be explored to extend these damage data. For example, in the fs and ps regimes, higher LIDT energies are expected due to lower thermal effects.

\section{CONCLUSION}

In this letter we have presented the first study to our knowledge of LIDT energies of nanofibers in air and in different liquids for several radii. The measurements were performed in the ns regime and for $\mathrm{cm}$ length nanofibers. A significant number of samples were tested, and the results we obtained were very repeatable. Immerging a nanofiber in a liquid enhanced the LIDT energy, the most significant increase having been obtained for nanofibers immersed in water: the LIDT energy has been almost multiplied by a factor of 2 compared with air. This study also showed that the use of liquids, in addition to providing a way to control the dispersion [14], offers a new degree of freedom to widen the field of applications of nanofibers, where high peak powers are needed [4].

\section{ACKNOWLEDGMENT}

The authors thank the French National Research Agency (ANR) for supporting M. Bouhadida's PhD. The authors thank Anne-Lise Coutrot (Laboratoire Charles Fabry, CNRS, France) for the MEB pictures of the nanofiber.

\section{REFERENCES}

[1] M. A. Foster, A.C. Turner, M. Lipson, A.L. Gaeta, "Nonlinear optics in photonic nanowires", Opt. Express 16, pp. 1300-1320 (2008).

[2] L. Tong, "Micro/Nanofibre optical sensors: Challenges and prospects", Sensors, 18, 903 (2018).

[3] K. S. Rajasree, T. Ray, K. Karlsson, J. L. Everett, and S. Nic Chormaic, "Generation of cold Rydberg atoms at submicron 
distances from an optical nanofiber", Phys. Rev. Research 2, 012038(R) February 2020.

[4] M. Bouhadida, J. C. Beugnot, P. Delaye, K. Phan Huy and S. Lebrun, "Highly efficient and reproducible evanescent Raman converters based on a silica nanofiber immersed in a liquid", Applied Physics B, 125 (12), pp.1-7 (sept. 2019).

[5] G. Fanjoux, J. Chrétien, A. Godet, K. Phan-Huy, J.C.Beugnot, T. Sylvestre, "Demonstration of the evanescent Kerr effect in optical nanofibers", Opt. Express 27 (20), pp. 29460-29470 (2019).

[6] A. M. Rubenchik and M. D. Feit, "Initiation, growth and mitigation of UV laser induced damage in fused silica," Proc.SPIE 4679, 79-95 (2002).

[7] A. V. Smith, B. T. Do, G. R. Hadley, R. K. Farraw, "Optical Damage Limits to Pulse Energy From Fibers", IEEE Journal of selected topics in quantum electronics, 15(1), pp. 153 (2009).

[8] N. Kuzuu, K. Yoshida, H. Yoshida, T. Kamimura, and N. Kamisugi, "Laser-induced bulk damage in various types of vitreous silica at $1064,532,355$, and $266 \mathrm{~nm}$ : evidence of different damage mechanisms between 266-nm and longer wavelengths", Appl. Opt. 38, pp. 2510 (1999).

[9] P. S. Hsu, A. K. Patnaik, J. R. Gord, T. R. Meyer, W. D. Kulatilka and S. Roy, "Investigation of optical fibers for coherent anti-Stokes Raman scattering (CARS) spectroscopy in reacting flows", Exp Fluids 49:969-984 (2010).

[10] C. Wuttke and A. Rauschenbeutel, "Thermalization via Heat Radiation of an Individual Object Thinner than the Thermal Wavelength”, Phys. Rev. Lett. 111, 024301, July 2013.

[11] A.W. Snyder, J. Love, "Optical Waveguide Theory", Chapt. 14, Kluwer Academic Publisher, Boston/Dortrecht/London, 2000.

[12] P. Anderson, S. Jalnapurkar, E. S. Moiseev, D. Chang, P. E. Barclay, A. Lezama and A. I. Lvovsky, "Optical nanofiber temperature monitoring via double heterodyne detection", AIP Advances 8, 055005 (2018).

[13] S. Yiou, S. Yiou, P. Delaye, A. Rouvie, J. Chinaud, R. Frey, G. Roosen, P. Viale, S. Février, P. Roy, J.- L. Auguste, and J.-M. Blondy, "Stimulated Raman scattering in an ethanol core microstructured optical fiber", Optics Express, 13(12), pp. 4786-4791 (2005).

[14] C. M. B. Cordeiro, W. J. Wadsworth, T. A. Birks and P. St.J. Russell, "Octave supercontinuum generated in tapered conventional fibres by a nanosecond $1064 \mathrm{~nm}$ laser", presented at Conf. Lasers Electro-Opt. (2004), paper CThC2.

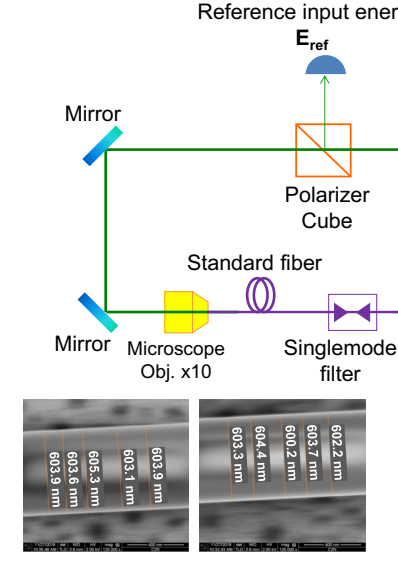

Reference input energy
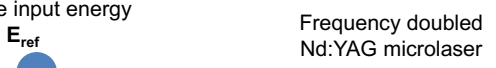
$\mathrm{Nd}$ :YAG microlaser

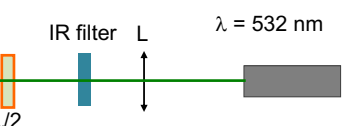

Fig. 1. Experimental setup. L: collimating lens. IR filter: infrared filter. Bottom left: MEB pictures of a NF2 sample (expected radius: $300 \mathrm{~nm}$ ) separated by 4 $\mathrm{mm}$. The single mode filter was used for SMF28 only. Bottom right: far-field picture of the output mode with cross section and Gaussian fit.

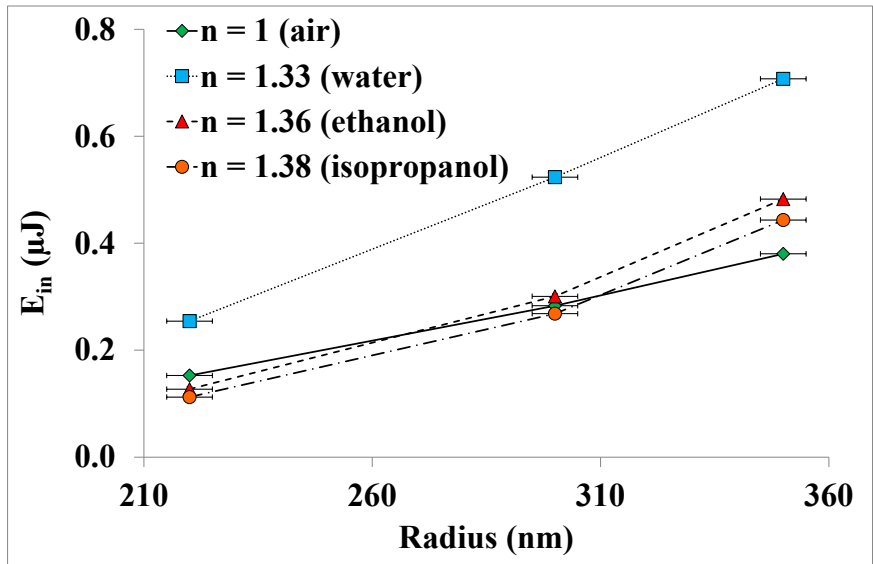

Fig. 2. Incident LIDT energy inside $\mathrm{E}_{\text {in }}(\mu \mathrm{J})$ versus nanofiber radius. Lines are guides for the eyes.

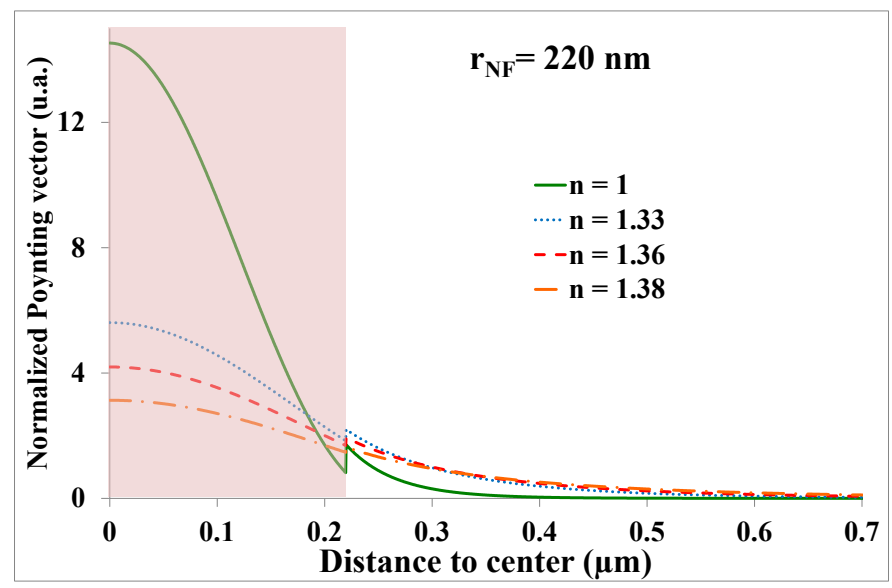

Fig. 3. Normalized Poynting vector Sz profile for a nanofiber radius of $220 \mathrm{~nm}$ for 4 different refractive indexes of the surrounding medium. The colored box shows the limit between the nanofiber and the surrounding medium.

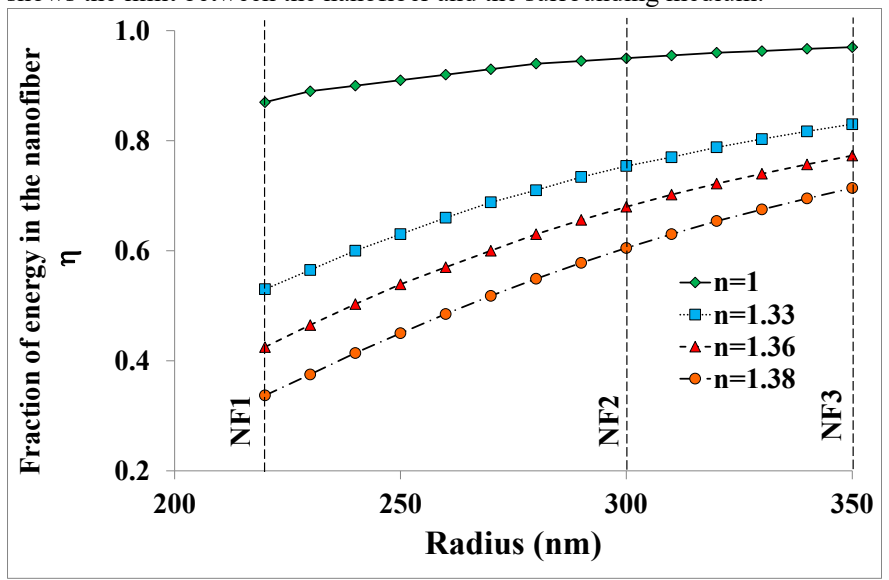

Fig. 4. Fraction of energy in the nanofiber versus the radius of the nanofiber for 4 different refractive indexes of the surrounding medium. Vertical dashed lines indicate the studied nanofibers NF1, NF2 and NF3. 